\title{
Cloning, structural modelling and characterization of VesT2s, a wasp venom hyaluronidase (HAase) from Vespa tropica
}

\author{
Prapenpuksiri Rungsa', Paroonkorn Incamnoi ${ }^{2}$, Sophida Sukprasert ${ }^{3}$, Nunthawun Uawonggul ${ }^{4}$, \\ Sompong Klaynongsruang ${ }^{1}$, Jureerut Daduang ${ }^{5}$, Rina Patramanon', Sittiruk Roytrakul ${ }^{6}$ and Sakda Daduang ${ }^{1,7^{*}}$
}

\begin{abstract}
Background: Wasp venom is a complex mixture containing proteins, enzymes and small molecules, including some of the most dangerous allergens. The greater banded wasp (Vespa tropica) is well-known for its lethal venom, whose one of the major components is a hyaluronidase (HAase). It is believed that the high protein proportion and activity of this enzyme is responsible for the venom potency.

Methods: In the present study, cDNA cloning, sequencing and 3D-structure of Vespa tropica venom HAase were described. Anti-native HAase antibody was used for neutralization assay.

Results: Two isoforms, VesT2a and VesT2b, were classified as members of the glycosidase hydrolase 56 family with high similarity (42-97\%) to the allergen venom HAase. VesT2a gene contained 1486 nucleotide residues encoding 357 amino acids whereas the VesT2b isoform consisted of 1411 residues encoding 356 amino acids. The mature VesT2a and VesT2b are similar in mass and pl after prediction. They are $39119.73 \mathrm{Da} / \mathrm{pl} 8.91$ and 39571.5 Da/pl 9.38, respectively. Two catalytic residues in VesT2a, Asp107 and Glu109 were substituted in VesT2b by Asn, thus impeding enzymatic activity. The 3D-structure of the VesT2s isoform consisted of a central core $(\alpha / \beta)_{7}$ barrel and two disulfide bridges. The five putative glycosylation sites (Asn79, Asn99, Asn127, Asn187 and Asn325) of VesT2a and the three glycosylation sites (Asn1, Asn66 and Asn81) in VesT2b were predicted. An allergenic property significantly depends on the number of putative $\mathrm{N}$-glycosylation sites. The anti-native HAase serum specifically recognized to venom HAase was able to neutralize toxicity of $V$. tropica venom. The ratio of venom antiserum was 1:12.

Conclusions: The wasp venom allergy is known to cause life-threatening and fatal IgE-mediated anaphylactic reactions in allergic individuals. Structural analysis was a helpful tool for prediction of allergenic properties including their cross reactivity among the vespid HAase.
\end{abstract}

Keyword: Wasp venom, Vespa tropica, Hyaluronidase (HAase)

\section{Background}

Vespidae venom consists of complex mixtures of enzymes, proteins, peptides and small molecules responsible for many of the non-allergic and mild allergic reactions - such as local pain, inflammation and itching as well as moderate and serious allergic reactions - such

\footnotetext{
* Correspondence: sakdad@kku.ac.th

${ }^{1}$ Protein and Proteomics Research Center for Commercial and Industrial Purposes (ProCCl), Department of Biochemistry, Faculty of Science, Khon Kaen University, Khon Kaen 40002, Thailand

${ }^{7}$ Division of Pharmacognosy and Toxicology, Faculty of Pharmaceutical

Sciences, Khon Kaen University, Khon Kaen, Thailand

Full list of author information is available at the end of the article
}

as anaphylaxis, and delayed hypersensitivity - including systemic toxic reactions, coagulopathy, acute renal failure and hepatotoxicity $[1,2]$. Wasp venom contains many biological active compounds [3, 4]. The major allergens are phospholipase A1, hyaluronidase (HAase) and antigen 5 [5-8].

Venom HAase is an enzyme that hydrolyses hyaluronic acid (HA), one of the primary components of the extracellular matrix of vertebrates, which facilitates venom toxin diffusion into the tissue and blood circulation of the prey $[9,10]$. HAase mainly acts as a "spreading factor" to enhance venom action. It has been 
identified in the venom of animals including snakes, bees, scorpions, fish, spiders, ants, wasps, caterpillars etc. [11-16]. Clinical studies have demonstrated that HAase is an "allergic factor" due to its ability to initiate pathogenic reactions in the majority of venom allergic patients [17-19]. It is also able to induce several anaphylactic IgE-mediated reactions in humans and has been suggested to be involved in the difficulties in the clinical diagnosis of venom allergic individuals [20-22]. The wasp venom HAase belongs to the hyaluronate glycanohydrolase family (EC 3.2.1.35), which degrades hyaluronic acid (HA) [23, 24]. Wasp venom HAase is responsible for the cross-reactivity of wasp and bee venom sera in patients as well $[2,25]$.

The greater banded wasp (Vespa tropica) is mostly distributed in the forest throughout Indochina peninsula including Thailand. It has a body length of up to $5 \mathrm{~cm}$ and its nest is usually found underground [26]. $V$. tropica is among the most venomous known insects. The lethal dose of its pure venom in experimental animals $\left(\mathrm{LD}_{50}\right.$ of approximately $2.8 \mathrm{mg} / \mathrm{kg}$ in mice) is more potent than that of $V$. affinis venom [26, 27]. The potency of $V$. tropica venom has been reported to nearly stop the end plate potentials of Drosophila larvae in nerve-muscle preparation in response to treatment with this venom [28]. HAase was reported to be a major protein in $V$. tropica venom, where it is found by 2.5 -fold the proportion observed in $V$. affinis venom [26]. The understanding of HAase in terms of biochemical and structural characterization of these wasps is important for the development of new tools for treating multiple stings and for diagnosis and therapy of allergic reactions caused by this venom. Therefore, the present study aimed to characterize HAase isoforms in the venom of $V$. tropica by analyzing its sequence and 3D modelling.

\section{Methods \\ Animals}

The wasps were collected from Siang Sao Village, Sri Songkram district, Nakorn Panom Province, northeastern Thailand [26]. The worker wasps were immediately shocked on ice. The venom reservoirs were removed from the sting apparatus by removing them from the bodies with forceps and squeezing. The droplets of venom and specimens of $V$. tropica were collected in a $1.5-\mathrm{mL}$ microcentrifuge tube and then keep at $-80{ }^{\circ} \mathrm{C}$ until use.

\section{RT-PCR and rapid amplification of CDNA ends ( $5^{\prime}$ and 3' RACE)}

Total RNA was extracted from the venom gland of $V$. tropica with TRIzol ${ }^{\circ}$ reagent (Invitrogen, Life technologies, USA). RT-PCR was performed using the RevertAid First strand cDNA synthesis kit (Thermo Scientific, USA) as described in the instruction manual. PCR primers for the amplification of VesT2 were designed based on the sequence similarity of the conserved region of HAase from vespid venom and conserved nucleotide sequences corresponding to peptide sequences obtained from LC-MS/MS analysis (Table 1) [26]. The PCR was performed using green master mix reagent kits with Taq DNA polymerase (Promega, Singapore). The reaction contained $2 \mu \mathrm{g}$ of cDNA, 1 UTaq DNA polymerase, $2.0 \mathrm{mM}$ dNTP, $2.0 \mathrm{mM}$ $\mathrm{MgCl}_{2}$ and $2 \mu \mathrm{M}$ of primerin to a final volume of $25 \mu \mathrm{L}$ under the following conditions: initial denaturation for 5 min at $94{ }^{\circ} \mathrm{C}$, followed by 35 cycles at $94{ }^{\circ} \mathrm{C}(30 \mathrm{~s}) ; 55^{\circ} \mathrm{C}$

Table 1 Primer design of gene-specific primers and PCR product size

\begin{tabular}{|c|c|c|}
\hline Forward primer & Reverse primer & Product size (bp) \\
\hline \multicolumn{3}{|l|}{ Full nucleotide sequence active form } \\
\hline F4 GCCAGACTTTTCATGGAGGA (GSP1 for active) & R3 (7) ATCAGGGGTCAGTTCACGTC (GSP1 for active) & 225 \\
\hline $\begin{array}{l}\text { Adaptor primer (AP) } \\
\text { 5'GGCCACGCGTCGACTAGTAC (T) } 16 \\
\text { (GSP for CDNA synthesis of 3' RACE system) }\end{array}$ & R4 (8) CGTCGGTCTCGGTAAGAAAA & \\
\hline Abridged universal amplification primer (AUAP) & R5 (9) GTTCTCGTGCATCGCTGTAA & \\
\hline $\begin{array}{l}\text { VesT2a (F) Ncol } \\
\text { CCATGGCTTCCGAGAGACC }\end{array}$ & $\begin{array}{l}\text { VesT2a (R) Xhol } \\
\text { CTCGAGTTAGTTAACGGCTTCTG }\end{array}$ & \\
\hline \multicolumn{3}{|l|}{ Full nucleotide sequence inactive form } \\
\hline $\begin{array}{l}\text { F1 CGAAAAGGAAGCGTCGAATA } \\
\text { (GSP for RT-PCR inactive form) }\end{array}$ & R1 CATCTTGTCGTTCTCGCTCA (GSP for RT-PCR inactive form) & 190 \\
\hline F2 CTTCGGCGTCTATTTCAAGG (GSP for RT-PCR inactive form) & R2CCGCTAAGACAGTGGGGATA (GSP for inactive form) & 229 \\
\hline $\begin{array}{l}\text { Adaptor primer (AP) } \\
5^{\prime} \text { GGCCACGCGTCGACTAGTAC (T) } 16 \\
\text { (GSP for CDNA synthesis of 3' RACE system) }\end{array}$ & R2 (1) CATCTTGTCGTTCTCGCTCA (GSP for RT-PCR inactive form) & \\
\hline Abridged universal amplification primer (AUAP) & R1 (2) CCGCTAAGACAGTGGGGATA (GSP for inactive form) & \\
\hline
\end{tabular}

The bold letters represent the restriction sites 
(30 s); $72{ }^{\circ} \mathrm{C}(1 \mathrm{~min})$ and a final extension at $72{ }^{\circ} \mathrm{C}$ for 5 min. The rapid amplification of cDNA ends (RACE) was performed with the RACE system (Invitrogen, Life Technologies, USA). The RACE PCR products were cloned into the $\mathrm{pGEM}^{\circ}-\mathrm{T}$ easy vector (Promega, USA) for sequencing [29].

\section{Sequence analysis and structure modelling}

The basic characterizations of the gene and protein sequences were analyzed using NCBI (http://www.ncbi.nlm. nih.gov/Database/index.html) and the basic local alignment search tool BLAST (http://www.ncbi.nlm.nih.gov/BLAST/). The phylogenic tree was created using CLUSTAL-X software analysis using the Neighbour-Joining method [30]. The three-dimensional models were created using the SWISS-MODEL program, the automated protein homology modelling template at ExPASY (Switzerland) and a template search with the Alignment Mode program from the protein database (http://swissmodel.expasy.org/) $[31,32]$. The model was elucidated as a PDB file, and the structure was previewed and analyzed using Swiss-Pdb Viewer Deep View v4 software (http://www.expasy.org/). The molecular mass and isoelectric points were computed using the Compute $\mathrm{pI} / \mathrm{MW}$ tool of ExPASy Bioinformatics (http://web.expasy.org/compute_pi/). The N-glycosylation sites were predicted using the CBS prediction severs (http://www.cbs.dtu.dk/services/NetNGlyc/) and compared with other wasp and bee venom HAases.

\section{Zymographic HAase activity assay}

The $V$. tropica venom HAase activity was detected using $10 \%$ SDS-PAGE containing hyaluronic acid as a substrate. Proteins were separated at $15 \mathrm{~mA}$. The gel was incubated in $3 \%$ Triton $\mathrm{X}-100$ for $1 \mathrm{~h}$ with agitation in order to remove SDS and then transferred to the HAase assay buffer $(0.15 \mathrm{M} \mathrm{NaCl}$ in $0.1 \mathrm{M}$ formate buffer), rinsed twice with assay buffer, and then incubated on a rotating shaker for $16 \mathrm{~h}$ at $37{ }^{\circ} \mathrm{C}$. The gels were rinsed twice with distilled water and stained in $0.5 \%$ Alcian blue solution for $1 \mathrm{~h}$. The destain was performed with $7 \%$ acetic acid that was changed every $1 \mathrm{~h}$ until clear bands appeared on a pale blue background [33].

\section{Turbidity HAase activity assay}

The turbidity HAase method followed the one by Pukrittayakamee et al. [34] with slight modifications. We mixed $0.5 \mathrm{mg} / \mathrm{mL} \mathrm{HA}$ and buffer containing $0.15 \mathrm{M}$ $\mathrm{NaCl}$ to a final volume of $100 \mu \mathrm{L}$ and incubated for $30 \mathrm{~min}$ at $37{ }^{\circ} \mathrm{C}$. The reaction was stopped using $200 \mu \mathrm{L}$ of $2 \%$ CTAB containing $2.5 \% \mathrm{NaOH}$. The absorbance was measured at $405 \mathrm{~nm}$. The turbidity reducing activity was expressed as the percentage of remaining HA by taking the absorbance of the tube at $100 \%$ in which no enzyme was added. The optimal $\mathrm{pH}$ of the venom HAase was determined by changing the buffers of the enzymatic turbidimetric venom HAase activity assay as follows: $0.2 \mathrm{M}$ formate buffer, $\mathrm{pH} 2-4 ; 0.2 \mathrm{M}$ acetate buffer, pH 5-6; 0.2 M Tris- $\mathrm{HCl}$ buffer, $\mathrm{pH}$ 7-10.

\section{Mouse anti-hyaluronidase serum}

The HAase band from zymographic gel were cut and frozen at $-70{ }^{\circ} \mathrm{C}$ overnight, the gel was freeze-dried and ground. Anesthetized mice were subcutaneously immunized with gel swollen in PBS buffer $(135 \mathrm{mM} \mathrm{NaCl}$, $1.5 \mathrm{mM} \mathrm{KH}_{2} \mathrm{PO}_{4}, 2.5 \mathrm{mM} \mathrm{KCl}$, and $8 \mathrm{mM} \mathrm{Na}_{2} \mathrm{HPO}_{4}$ ) emulsified with Freud's complete adjuvant. Mice were four times boosted with the antigen emulsified with incomplete Freund's adjuvant. After retro-orbital plexus bleeding, blood was kept at $4{ }^{\circ} \mathrm{C}$ for $12 \mathrm{~h}$ and centrifuged at $10000 \times g$ for antiserum collection.

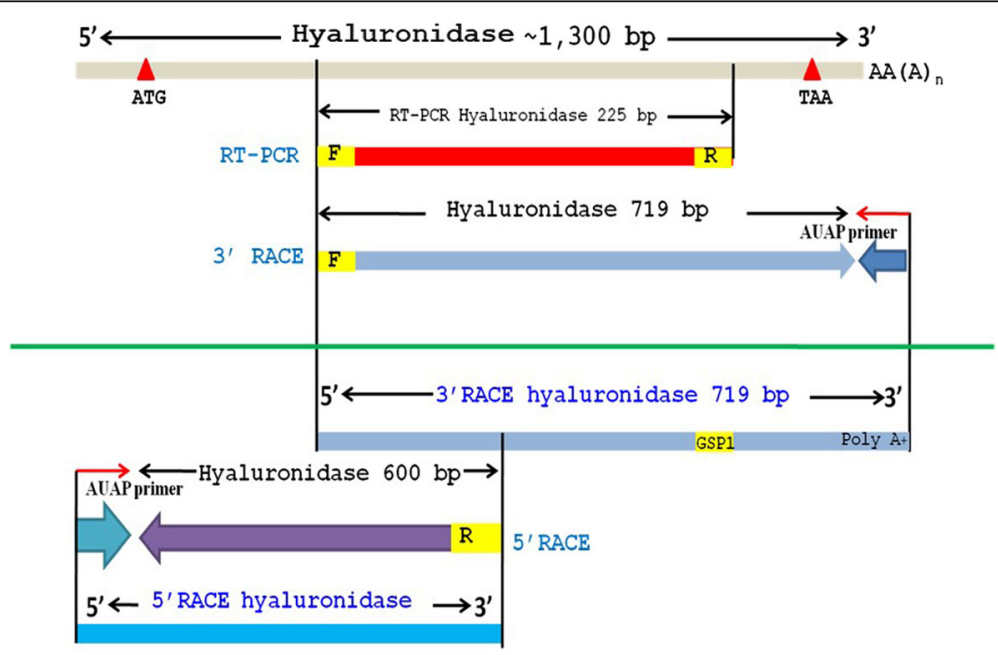

Fig. 1 The cloning strategies of Vespa tropica HAase (VesT2s). The 357 amino acid sequence of VesT2s was deduced by overlapping of the VesT2s HAase gene and determined by using RT-PCR and RACE-PCR 


\section{Western immunoblotting}

Proteins were separated by SDS-PAGE and blotted onto a nitrocellulose membrane (Bio-Rad, USA). After being eletrotransferred, the membrane was incubated with $5 \%$ nonfat dry milk for $1 \mathrm{~h}$, anti-HAase antibody for $1 \mathrm{~h}$ and goat anti-mouse IgG linked alkaline phosphatase (1:500) for $1 \mathrm{~h}$. The blotted bands were detected by a substrate kit (GE Healthcare, Sweden). The membrane was intensive washed before the next incubation.

\section{Neutralization assay}

Crickets (Gryllus sp.) were abdominally injected with venom pre-incubated with anti-HAase serum $10 \mathrm{~min}$ before considered paralyzed. The paralyzed crickets were defined as those that could return from the overturned position.

\section{Results}

Sequence and structural modelling analysis of VesT2s

The completed cDNA sequence was designed according to the peptide sequences obtained by LC-MS/MS and the sequence similarities of the conserved region of the other wasp venom hyaluronidases [26]. The primers were designed from nucleotide sequences based on the conserved region corresponding to the peptide sequence. The nucleotide fragment was obtained via RT-PCR. The

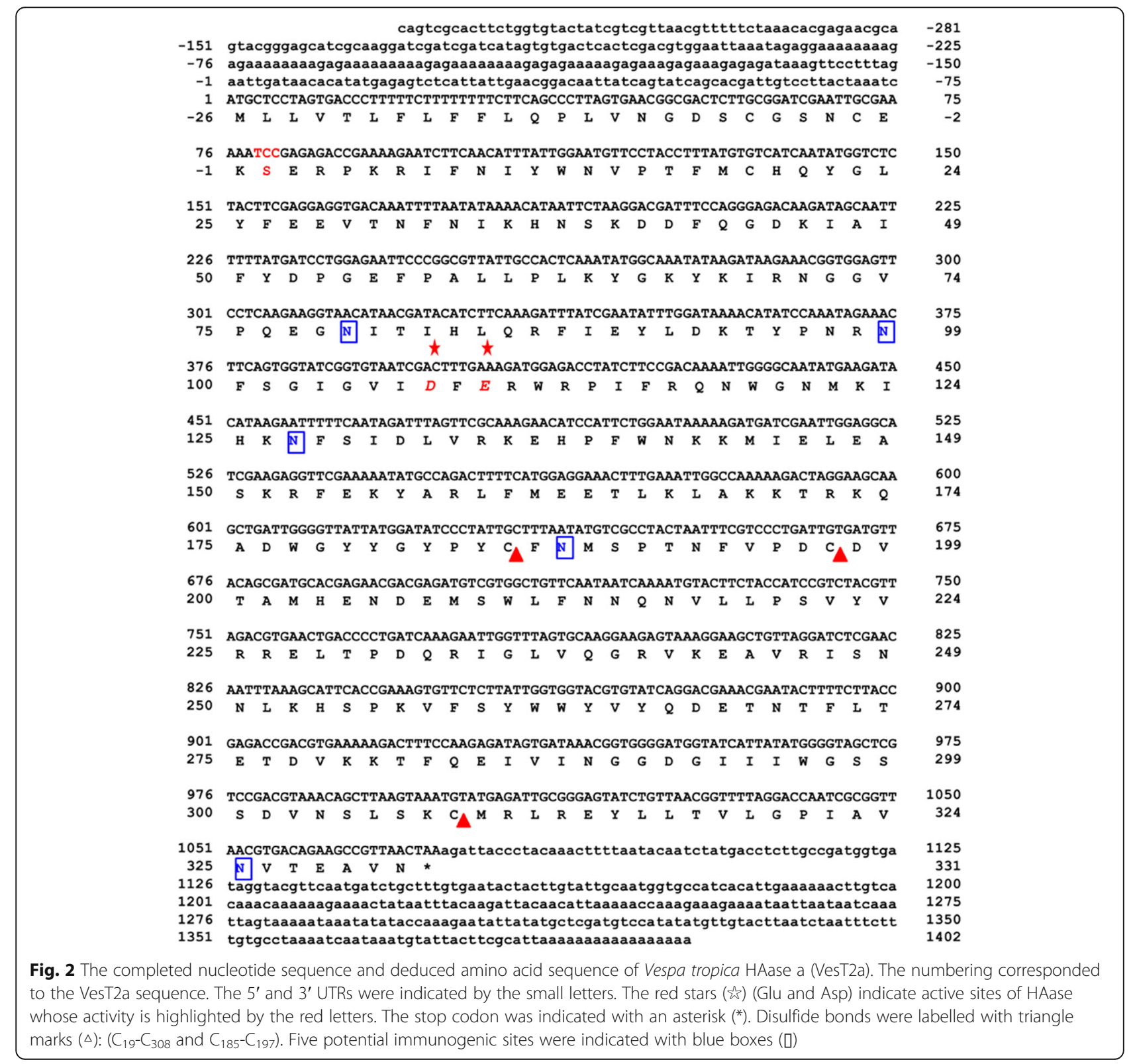


$3^{\prime}$ and $5^{\prime}$ end were determined using RACE. They were completely overlapped (Fig. 1). Two HAase isoforms, VesT2a and VesT2b, were obtained.

The full length VesT2a cDNA was 1,683 bp in length and contained $281 \mathrm{bp}$ of the $5^{\prime}$-untranslated region (UTR) and $328 \mathrm{bp}$ of the 3 '-UTR; 1,074 bp of an open reading frame (ORF) encoded a protein of 357 amino acids (Fig. 2). The primary sequence of the deduced VesT2a contained 357 amino acid residues including a predicted signal peptide (26 amino acid residues) that was rich in the amino acids Asn, Lys, Ile and Leu, with a predicted mature $\mathrm{pI}$ and molecular mass of 8.91 and 39,119.73 $\mathrm{Da}$, respectively. The five potentially immunogenic $N$-glycosylated sites (Asn-Xaa-Thr/Ser, where Xaa is any amino acid residue except proline) on residues Asn79, Asn99, Asn127, Asn187 and Asn325 were predicted. The two disulfide bridges (Cys19-Cys308 and Cys185-Cys197) were responsible for the stabilization of protein structure (Fig. 2).

Additionally, a putative HAase isoform was recently suggested as another component appearing in the 2DPAGE profile from the corresponding cDNA of VesT2b [26]. It had an experimental mass of approximately 46 to $47 \mathrm{kDa}$. After amplification using several strategies (Fig. 1), the VesT2b precursor contained a 195-bp 5'UTR, a 145-bp 3'-UTR and an 1146-bp ORF. The ORF consisted of a 57-bp predicted signal sequence, which corresponded to 19 amino acid residues, and a 1089-bp mature sequence encoding 337 amino acids. The primary sequence of the deduced VesT2b mature protein contained 337 amino acid residues (996 bp) and was rich in the amino acids Lys, Asn and Ile with a theoretical pI

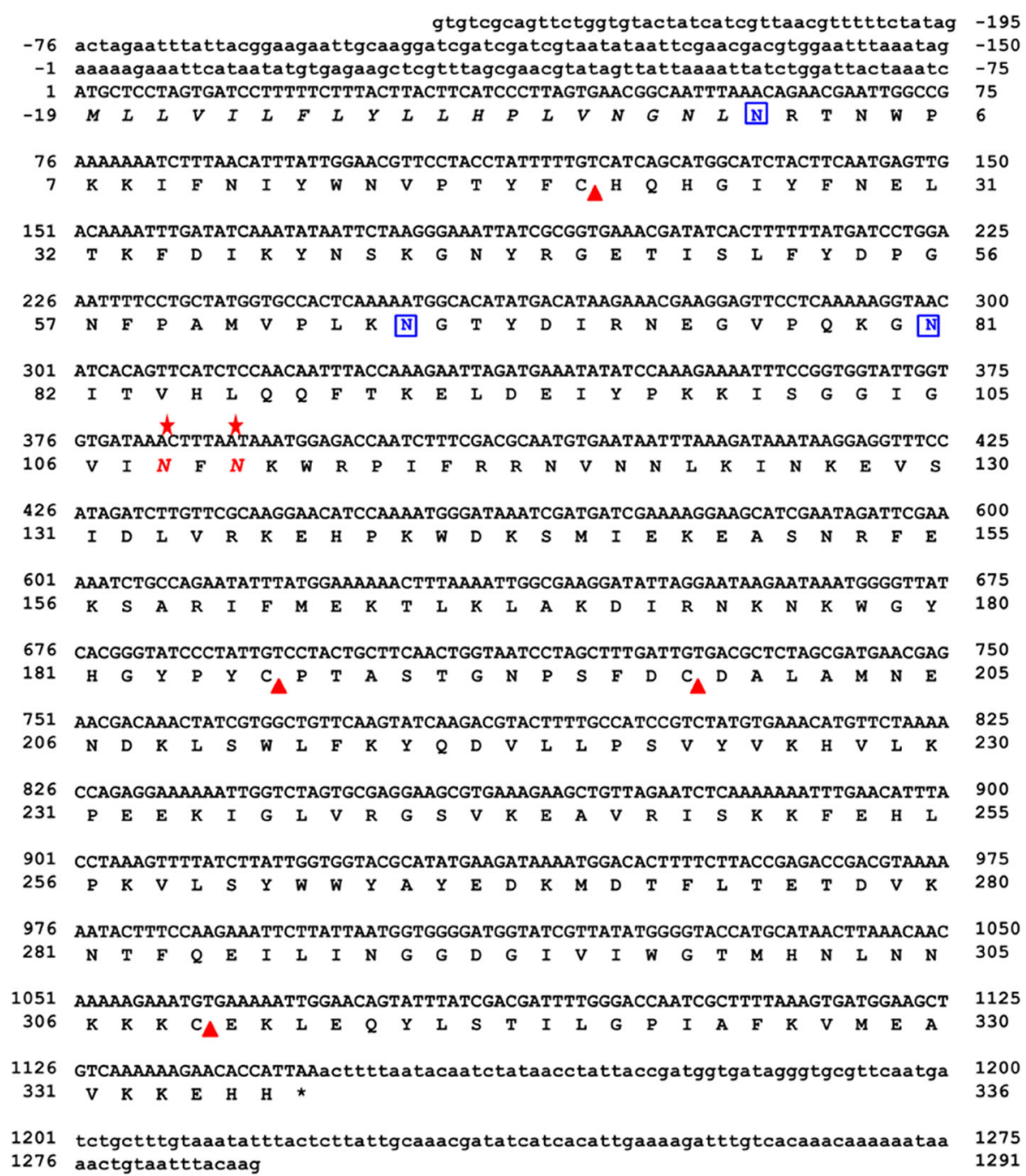

Fig. 3 The completed nucleotide sequence and deduced amino acid sequence of Vespa tropica HAase b (VesT2b). The numbering corresponded to the VesT2b sequence. The 5' and 3' UTRs were indicated by the small letters. Disulfide bonds were labelled with triangle marks $(\Delta)$ : $\left(C_{21}-C_{310}\right.$ and $\left.C_{187}-C_{199}\right)$. Three potential immunogenic sites were indicated with blue boxes ( $\mathrm{\square}$ ) 
Vest $2 \mathrm{a}$ VesT $2 b$ Vesv2a vesv $2 a$ Vesv2b VesG2a VesG 2 b Vesma2 DolM2 POIA2 Polp2 Api_M2

Vest $2 a$ Vest $2 b$ Vesv2a Vesv2b VesG2a VesG2b Vesma2 Dolma PolA2 Po1P2 Api_M2

Vest $2 a$ Vesv2a Vesv $2 b$ VesG2a VesG2b vesma2 Dolm2 PolA2 PolP2 Api_M2

Vest $2 a$ VesT 2b Vesv2a Vesv $2 b$ VesG2a Dolm2 PolA2 PolP2

Api_M2

Vest $2 a$ Vest 2b vesv2a Vesv $2 b$ VesG2a VesG $2 b$ Vesma2 Dolm2 DolM2 POIA2 Api_M2

Vest $2 a$ Vest 2b Vesv2a Vesv2b VesG2a VesG2a VesG $2 \mathrm{~b}$ VesMa Dolm2 POIA2 POIP2 Api_M2

vest2a Vest $2 \mathrm{~b}$ Vesv2a Vesv2b VesG2a VesG2b Vesma2 Dolm2

Po1A2

Polp2

Api_M2
---MLLVT LFLFFLQPLVNGDSCGSNCEKSER PKRI FN IYWNVPTFMCHQYGLYFEEVTN $-------M L L V I L$ FL YLLH PLVNGNLNRT NW PKKI FN I YWNVPTY FCHQHG IY FNELTK $--------------------------S E R$ PKRVFN IYWNVPTFMCHQYDLYFDEVTN $----------------------D R T$ IW PKKG FS IYWN I PTH FCHNFGVY FKELKQ --SERPKRVFNIYWNVPTFMCHQYDLYEDEVTN

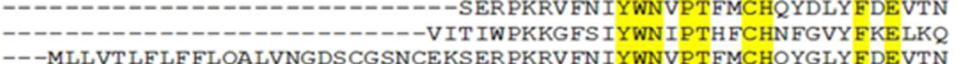
---MLLVT LF LF FL QALVNGDSCGSNCEKSER PKRV FN IYWNVPTFMCHQYGLY FDEVTN YVSLSPDSVENI ITDDISHQILSR SNCER SKR PKRVES IYWNVPTFMCHQYGMNFDEVTD Y

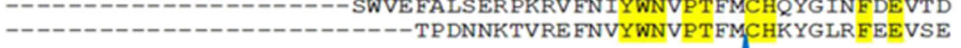

FN-I KHNSKDDFQGDK IA IFYDPGEF PALL PLKY GKYK IRNGGVPQEGNI T I HLQRF IEY FD-I KYNSKGNYRGET IS LFYD PGNF PAMV PLKNGTYDIRNE GV PQKGNI TVHLQQETKE FN-I KRNS KDDFOGDK IA IFYDPGEF PALLSLKDGKYKKR NGGV PQEGNI T I HLOKF IEN FN-I KYNSMNNFRGET IS LFYD PGNE PSMVLLKNGTYE IRNEGV PQKGNLTI HLEQETKE FN-IKRNSKDDFQGDKIAIFYD PGEF PALLSLKD GKYKKRNGGVPQEGNITI HLQKF IEN FN-I KYNSMNNERGET ISLFYDPGNF PSMVLLKNGTYE IRNEGVPQKGNLTI HLEQFTKE FN-I KHNS KDNEQGDKIA IFYD PGEF PALL PLNY GKYK IRNGGV PQEGNI T I HLQRF IEY FN-I KHNSKDDFOGDK IS IFYDPGEFPALL PLKEGNYK IRNGGVPQEGNI T I HLQRE IEN FN-I KHNSKDNFRGET IS IYYDPGKF PALMPL KNGNYEERNGGVPQRGNI TI HLQQFNED FN-I KHNSKDNFRGET LA IYYD PGNF PALI PVNNGKYKERNGGVPQRGNI TI HLQQFNED KY GI LQNWMD KFRGEE IA IL YDPGMF PALLKD PNGNVVARNGGVPQLGNLTKHLQVERDH

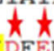

LDKTYPNRNE SGIGVIDFERWRPI FRONWGNMKI HKNFSI DLVRKEHP FWNKKM I ELEAS LDE I YPKK IS GG IGVINFNKWR PI FRRNVNNLKINKEVSI DLVRKEHPKWDKSMI EKEAS LDKI YPNRNE SGIGVI DFERWR P I FRQNWGNMKI HKNESI DLVRNEHP TWNKKM I ELEAS LDE I YPKK IA GG I GV I HFHNWR P I FRRNVD NL K I NKD I S I DLVRKEHP KWDKSM I EKEAS LDKTYPNRNF SGI GVI DFERWR P I FRQNWGNMKI HKNESI DLVRNEHP TWNKKM I ELEAS LDE I YPKK IA GG I GVI HFHNWR P I FRRNVDNLKI NKD I S I DLVRKEHPKWDKSM I EKEAS LDKT YPNRNF SGIGVI DFERWR P I FRQNWGNMKI YKNES I DLVRKEHP FWNKKM I ELEAS LDKTYPNRNFNG I GVI DFERWRP I FRONWGNMMI HKKES I DLVRNEHP FWDKKM I ELEAS LDKMTP DKNE GG I GVI DFERWKPI FRQNWGNT I HKKY I ELVRKEHP KWSESM I EAEAT LDKMTPDKNF GGIGVI DFERWKPI FRQNWGNTEI HKKY SIELVR YEHP KWSESM I EAEAT LINQ I PDKSF PGVGVI DFESWR PI FRQNWASLQPYKKLSVEVVRREHP FWDDQRVEQEAK

KRFEKYARLFMEETLKLAKKTRKOADWGYYGY PYCFNMSPTNFVPDCDVTAMHENDEMSW NR FEKSAR IFMEKTLKLAKD IRNKNKWGYHGYPYCPTAST GNPS FDCDALAMNENDKLSW KR FE KYAR FFMEET LKLAKKTRKQADWGYYGY PYCFNMSPNNLVPECDVTAMHENDKMSW NR FE TSAK I FME KT LKLAKE IRKKTEWGYHGY PHCLSGST DKPS FDCDALSMSENDKMSW KR FE KYAR FFMEETLKLAKKTRKQADWGYYGY PYCENMSPNNLVPECDVTAMHENDKMSW NR FE TSAK I FME KT LKLAKE IRKKTEWGYHGY PHCLSGST DKPS FDCDALSMSENDKMSW KR FEKYARLFMEETLKLAKKTRKOADWGYYGY PYCFNMSPTNFVPDCDVT ARDENNEMSW KRFE KYARLFMEET LKLAKKTRKQADWGYYGY PYCFNMSPNNLVPDCDATAMLENDKMSW
KKFEKYARYFMEET LKLAKKTRKRAKWGYYGFPYCYNVTPNN PQDCDAKAT IENDRLSW KKFEKYARLFMEETLKLAKKTRKRAKWGYYGF PYCYNYTPNN PGPDCDAKAMIENDRLSW RR FEKYGQLFMEET LKAAKRMR PAANWGYYAY PYCYNLTPNQPSAQFEATTMQENDKMSW

LENNQNVLLPSVYVRRELTPDQRI GLVQGRVKEAVRISNNLKHSP-KVES YWWYVYQDET LFKYODVLIPSVYVKHVL KPEEKIGLVRGSVKEAVR ISKKEEHLP-KVLSYWWYAYEDKM LFNNQNVLLPSVYVRQELTPDQRI GLVQGRVKEAVRISNNLKHSP-KVLSYWWYVYQDET LFNNQNVLLPSVYVRQELTPDQRI GLVQGRVKEAVR ISNNLKHSP-KVLSYWWYVYQDET LFNNQNVLLP SVYVRQELTP DQR I GLVQGRVKEAVR ISNNLKHSP-KVLS YWWYVYQDET LFNNQNVLLPS I YLKNVLKP DEKI HLVQERLKEA IR I SKNLKHLP-KVLPYWWYTYQDKE LFNNQNVLLP SVYIRRELTPDQRI GLVQGRVKEAVR ISNKLKHS P-KVFS YWWYVYQDET LFNNQNVLLP SVYIRHELTP DQRVGLVQ GRVKEAVR ISNNLKHS P-KVLS YWWYVYQDDT MYNNOE ILEP SVYVRHEOKPEERVYLVOGRIKEAVR ISNNLEHSP-SVLAYWWYVYODKM MYNNQEILEPSVYVRHELTPDQRVYLVQGRIKEAVRISNNLKHSP-KVISYWWYVYQDKM LFESEDVLLPSVYLRWNLTSGERVGLVGGRVKEALRIAROMTTSRKKVLPYYWYKYODRR

NT FLTETDVKKT FQEIVINGGDGI I IWGSSSDVNSLSKCMRLREYLLTVLGPIAVNVTEA DT FLTE TDVKNT FQEI L INGGDGIVIWGTMHNLNNKKKCEKLEQYLST ILGP IA FKVMEA NT FLTETDVKKT FQEIVINGGDGI I IWGSSSDVNSLSKCKRLQDYLLTVLGP IA INVTEA SI FLTEADVKNT FKEI LTNGADGI I IWGVS YELT DRKRCEKLKE YLMK I LGP IAFKVTKA NTYLTE TDVKKT FQEIVINGGDGI I IWGSS SDVNSLSKCKRLQDYLLTVLGP IAVNVTEA SI FL TEADVKNT FKE I LTNGADGI I IWGVSYE LT DRKRCE KL KE YLMK I LGP------NT FLTETDVKKTFOEIVINGGDGI I IWGSSSDVNSLSKCTRLREYLLTVLGPIAVNVTEA NTLTE TDVKTTQQIVINGGDGIIIWGSSSDVNSLSKCTRLRE YLITVGPIAVNVTEA NT FLTETDVKKTFQEIAINGGDGIIIWGSSSDVNSLSKCKRLREYLLTVLGP ITVNVTET DI YLSETDVEKT FQEIVTNGGDGI I IWGSSSDVNSLSKCKRLRE YLLNTLGP EAVNVTET DTDLSRADLEATLRKITDLGADGF I IWGSSDDINTKAKCLQFREYLNNELGPAVKRI ALN

\begin{tabular}{|c|c|}
\hline 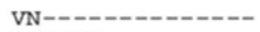 & $100.00 \%$ \\
\hline VKKEHH --------- & 61.52 z \\
\hline $\mathrm{VN}--------------$ & $93.05 \%$ \\
\hline VKENTPLNF------- & $57.35 \%$ \\
\hline VN---D---- -- -- - & $93.35 \%$ \\
\hline - - - - & $58.82 \%$ \\
\hline 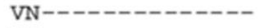 & $96.64 \%$ \\
\hline 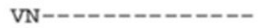 & 92.44 \% \\
\hline VNGRSSLNE------- & 69.75 ช \\
\hline 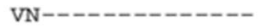 & 73.048 \\
\hline NNANDRLTVDVSVDQV & 41.14 ह \\
\hline
\end{tabular}

Fig. 4 (See legend on next page.) 
(See figure on previous page.)

Fig. 4 Sequence alignment of the deduced amino acid sequence of Vespa tropica HAase with other allergen venom HAases. VesT2s was aligned with the known HAases; VesV2a and VesV2b (Vespula vulgaris; active and inactive forms), VesG2a and VesG2b (Vespula germanica; active and inactive forms), Vespa magnifica (VesMa2), DolM2 (Dolichovespula maculata), PolA2 (Polistes annularis), PolP2 (Polybia paulista) and Api_M2 (Apis mellifera). The shaded yellow alignment corresponds to conserved residues in HAase. The N-terminus was shown in the underlined amino acid sequence obtained by Edman sequencing. The catalytic residues ( $D$ and $E$ letters) are indicated with the red stars ( $\hbar$ ) ). The conserved cysteine positions among the HAase are indicated with blue triangles $(\Delta)$

9.38 and a predicted molecular mass of $39571.5 \mathrm{Da}$. The three potentially immunogenic $N$-glycosylated sites (Asn1, Asn66 and Asn81) and the two disulfide bridges (Cys21-Cys310 and Cys187-Cys199) were predicted (Fig. 3). The VesT2s mature amino acid sequence in these studies had $61.52 \%$ homology; the two catalytic residues in VesT2a, Asp107 and Glu109, were substituted byAsn in VesT2b (Fig. 3).

The multi-sequence alignment of venom HAases (Fig. 4) showed the highest BLAST homology score ( $>90 \%$ identity) of VesT2a to many HAases, VesV2a of Vespula vulgaris, VesG2a of Vespa germanica, VesMa2 of Vespa magnifica, and Dol m 2 of Dolichovespula maculata, suggesting high evolutionary conservation among these species. The catalytic residues (Asp107 and Glu109) were conserved in active venom VesT2a $[20,22,35,36]$. The phylogenetic tree analysis revealed the highest similarity of VesT2a to VesMa2, which was higher than that of the VesT2b depicted in the phylogenetic tree of the insect HAase (Fig. 5). VesT2s contained cysteine residues that were conserved among venom HAases and also formed two disulfide bonds (Fig. 6a and b). For VesT2a, these were Cys19-Cys308 and Cys185-Cys197, whereas they were Cys21-Cys310 and Cys187-Cys199 in the structure of VesT2b.

VesV2 (PDB ID: 2ATM) was used as a template for computational homology modelling. VesT2a and VesT2b showed $92.28 \%$ and $62.69 \%$ sequence identity to VesV2,

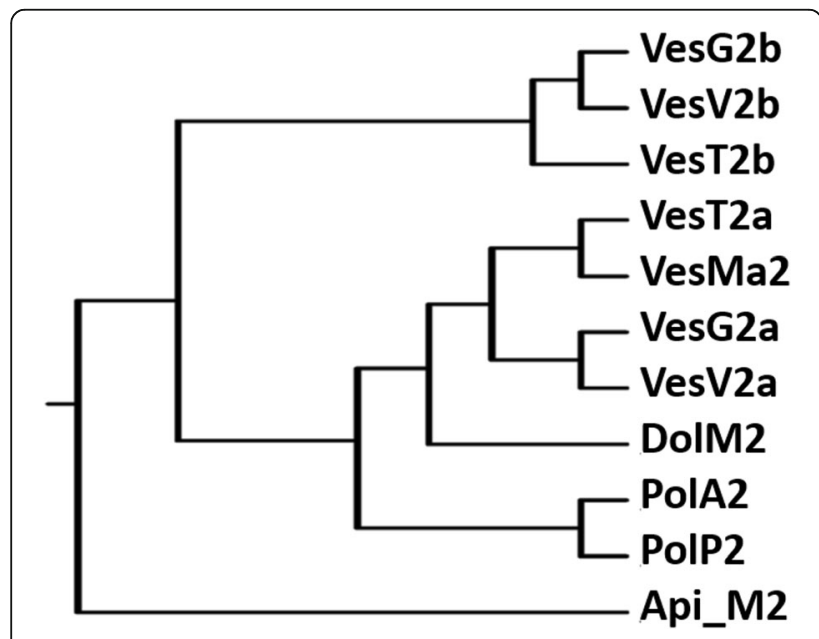

Fig. 5 The phylogenic tree of HAases from insect venoms respectively, with an $\mathrm{E}$ value of $6.19 \mathrm{e}^{-153}$. Based on the model, VesT2a and VesT2b displayed a central core $(\alpha /$ $\beta)_{7}$ consisting of seven helices and seven beta-sheets belonging to family 56 of glycoside hydrolases [37] (Fig. 7).

\section{HAase activity of wasp venom VesT2a}

$V$. tropica venom VesT2a was tested for specific HAase activity using zymographic method at $37{ }^{\circ} \mathrm{C}, \mathrm{pH} 3.7$, under reducing conditions. The result showed a transparent band (Fig. 8a). The turbidity method was used to determine the optimal $\mathrm{pH}$ of venom HAase, with hyaluronic acid as substrate. The V. tropica HAase, VesT2a, had an optimal $\mathrm{pH}$ of about 3 (Fig. 8b). It clearly displayed a higher HAase activity between $\mathrm{pH} 2$ and 5 .

\section{Neutralization assay}

The Western immunoblotting revealed the specificity of antibodies to their antigens when the titer was 1:100 (Fig. 9). The anti-HAase serum was able to reduce venom toxicity (Table 2). Non-paralyzed crickets were observed at the ratio 1:12 (venom: antiserum).

\section{Discussion}

In this study, we described the identification, biochemistry, bioactivity and structural characteristics of the HAase from the venom of greater banded wasp V. tropica. This study describes the existence of two isoforms of VesT2s, VesT2a and VesT2b. The primary sequence of VesT2a and VesT2b were clearly isoenzymes with $61.52 \%$ similarity but with different molecular masses and pIs of the mature sequence (357 amino acids/ $39119.73 \mathrm{Da} / \mathrm{pI} 8.91$ and 337 amino acids/39571.53 Da/

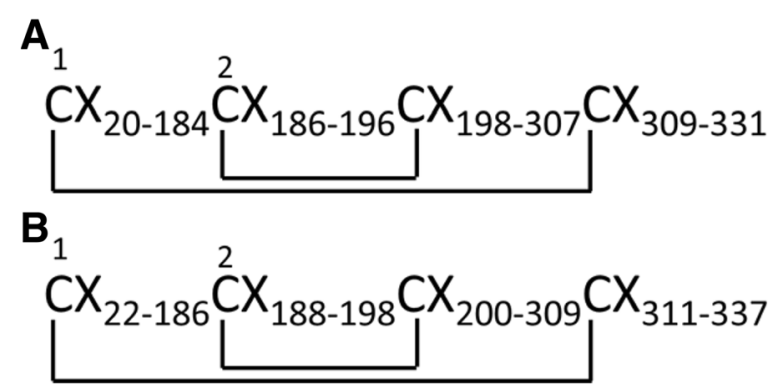

Fig. 6 The disulfide linkages of VesT2s [(a); VesT2a and (b); VesT2b] The two disulfide bridges (VesT2a; $C_{19}-C_{308}$ and $C_{185}-C_{197}$, VesT2b; $\mathrm{C}_{21}-\mathrm{C}_{310}$ and $\mathrm{C}_{187}-\mathrm{C}_{199}$ ) are linked via a solid line 

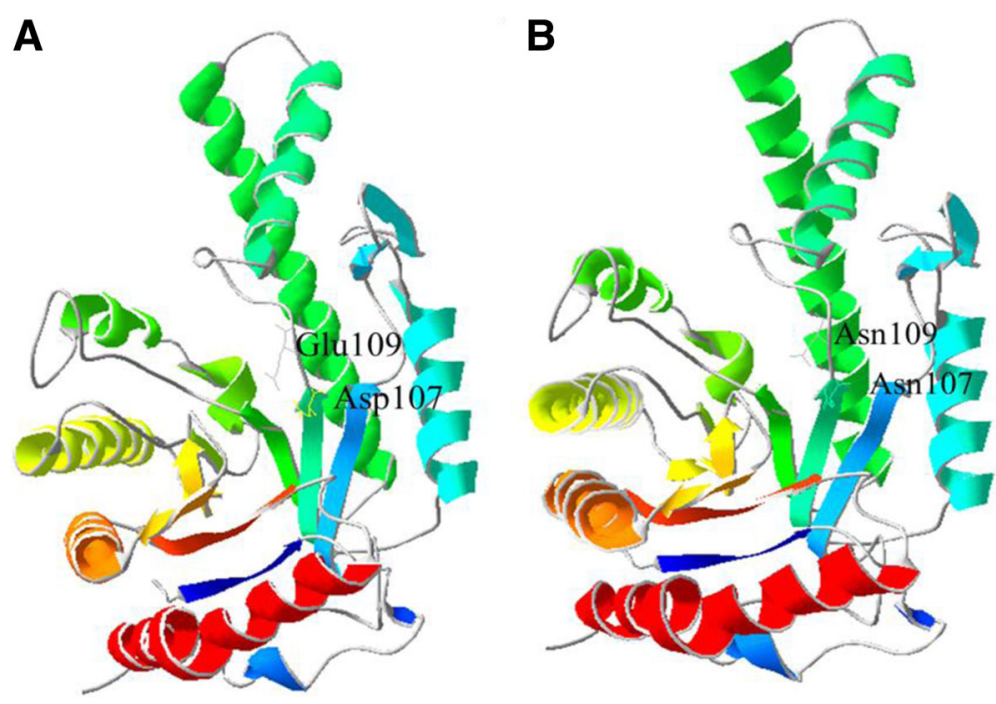

Fig. 7 The predicted three-dimensional structural modelling of VesT2s. The Vespa tropica HAase [VesT2a (a) and VesT2b (b)] modelling used VesV2 as a template (Vespula vulgaris, PDB accession number 2ATM_A). VesT2s was generated with SWISS-MODEL automated software and was visualized by the Swiss-Pdb Viewer Deep View v4.0 program. The two catalytic sites of VesT2a (Glu109 and Asp 107) were changed to Asn in VesT2b

pI 9.38, respectively). Mass differences were mainly estimated from amino acid variations, including the degree of glycosylation of VesT2s. However, they were classified into the same family of glycoside hydrolase family 56 by sequence similarity. This phenomenon also occurs with HAases in many species, such asVesV2a and VesV2b, the HAase isoenzymes in Vespula vugaris venom. VesV2a and b share $58 \%$ amino acid identity to each other $[5,20]$.

Rungsa et al. [26] indicated that the mass of HAase in $V$. tropica venom was approximately $43 \mathrm{kDa}$ after analysis by denaturing two-dimensional electrophoresis, which was confirmed by peptide mass fingerprinting. However, the mature sequence of HAase in this study, VesT2s, was smaller in size, with approximately $39 \mathrm{kDa}$. The molecular mass of about $43 \mathrm{kDa}$ of native VesT2s was not surprising, since wasp venom HAase is a glycoprotein whose differences in estimated values of theoretical pI and molecular masses are frequent $[9,38,39]$.

The phylogenetic tree demonstrated that VesT2a is found in the same cluster of active HAase from insect venoms. VesT2b is also found in a cluster of inactive
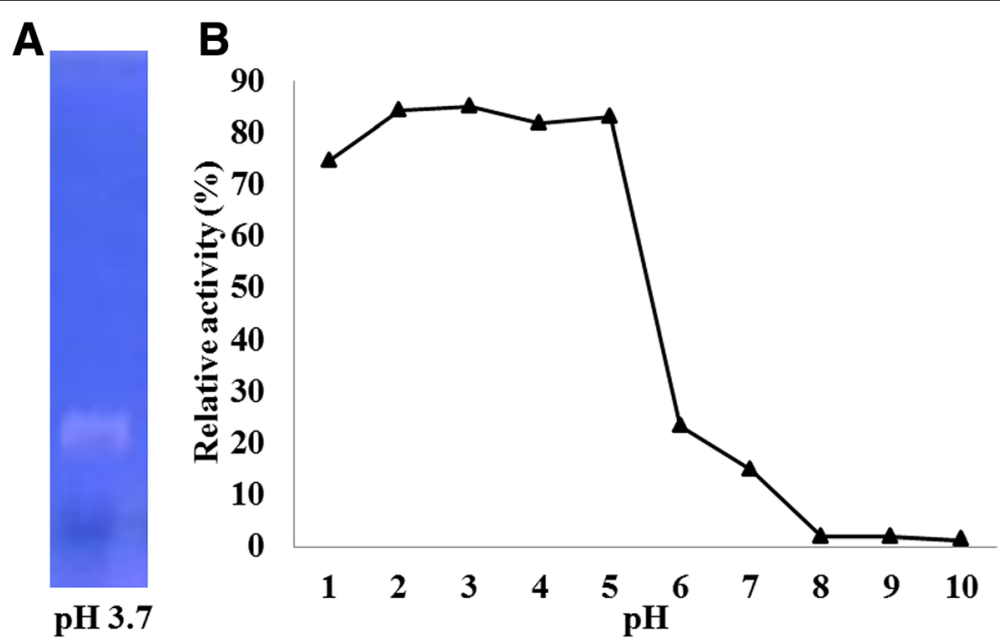

Fig. 8 The HAase activity. VesT2a: V. tropica venom was tested for specific HAase activity by the (a) zymographic method and (b) optimal pH using turbidimetric assay. For the zymogram HAase activity assay, samples were analysed using $10 \%$ SDS-PAGE containing hyaluronic acid under reducing conditions. The zymgraphic gel was developed overnight at $37^{\circ} \mathrm{C}$ under $\mathrm{pH} 3.7$. For the turbidimetric assay, the enzymatic activity of VesT2a, V. tropica venom was tested at various pHs using hyaluronic acid as a substrate at $37^{\circ} \mathrm{C}$ 


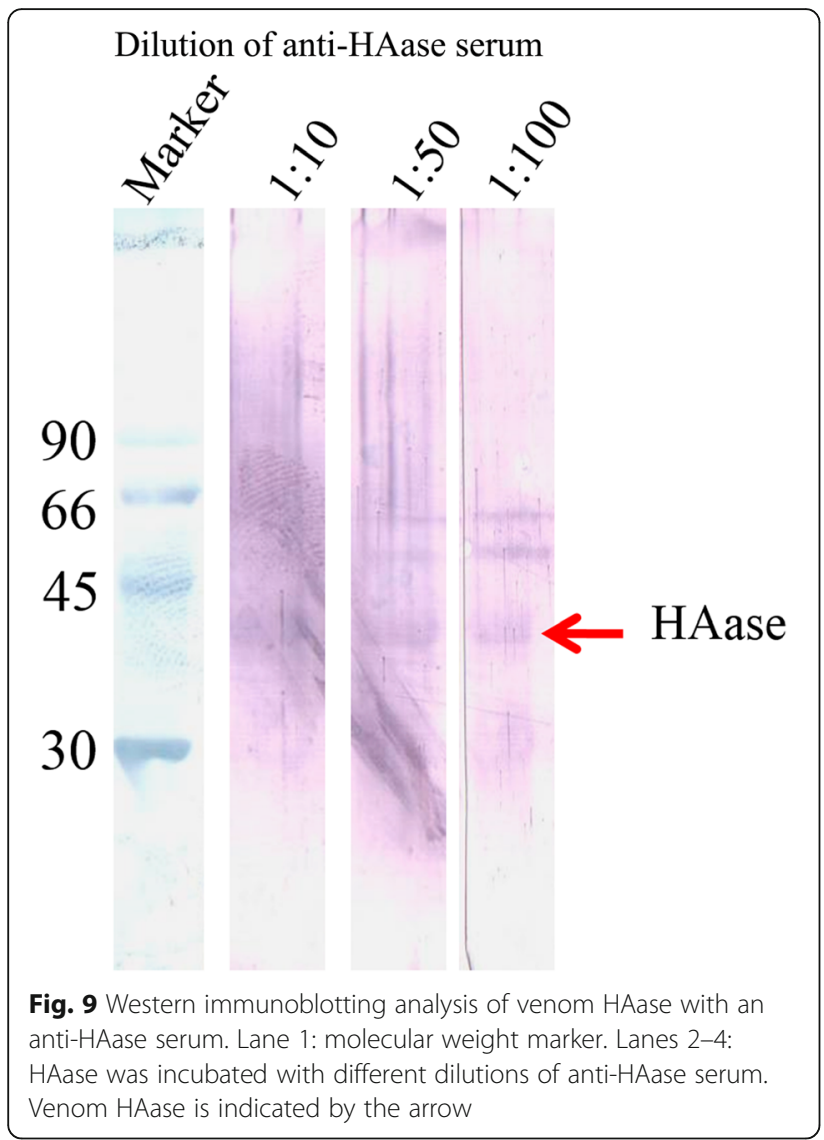

HAase from insect venoms $[2,20,35,38,40]$. The enzyme function of VesT2s is different because of two catalytic residues in VesT2a, Asp107 and Glu109. Both are substituted by Asn in VesT2b that has no HAase enzymatic activity towards various substrates [20, 35, 41]. The less acidic Asn cannot act as a proton donor as the acidic amino acids, Asp and Glu [36, 37].

Glycosylation sites are the most common posttranslational modification of many insect venom proteins as they contribute to biological activity, immunogenicity, and solubility, stability and protease resistance. VesT2s represents one of the strongest conserved hymenoptera venom allergens in wasps, yellow jackets and honeybees $[42,43]$. VesT2a is highly similar to VesMa2 (Vespa magnifica HAase) while VesT2b is close to VesV2b (Vespula vugaris HAase b). $V$. vugaris and $V$. magnifica also belong

Table 2 The neutralization assay of $V$. tropica venom against anti-HAase serum in crickets (Gryllus sp.)

\begin{tabular}{ll}
\hline $\begin{array}{l}\text { V. tropica venom: Anti-HAase } \\
\text { serum }(\mu \mathrm{L} / \mu \mathrm{L})\end{array}$ & $\begin{array}{l}\text { Neutralized crickets/total crickets after } \\
\text { injections with } V . \text { tropica venom and } \\
\text { anti-HAase serum }\end{array}$ \\
\hline $1: 4$ & $2 / 4$ \\
$1: 8$ & $1 / 4$ \\
$1: 12$ & $0 / 4$ \\
\hline
\end{tabular}

to the Vespidae family $[20,35,40]$. Therefore, we presume that the VesT2s isoform might have a similar structure and allergic properties.

Insect venom allergies are known to cause lifethreatening and sometimes fatal IgE-mediated anaphylactic reactions in allergic individuals. Approximately 30 to $50 \%$ of patients with insect venom allergies have IgE antibodies that react with both honeybee and yellow jacket venom [44]. Previous studies have demonstrated that human IgE antibodies share cross reactive B-cell epitopes with various venom HAases to $\mathrm{VesV} 2[2,25]$. Honeybee and yellow jacket venom HAases with a molecular mass of approximately $42-45 \mathrm{kDa}$ are considered to be major allergen proteins and are responsible for cross-reactivity with allergen patient sera [44]. The venom HAase in insects are classical allergens responsible for cross-reactivity. Nevertheless, the cross-reactivity of venom HAase was identified by cross reactive carbohydrate determinants (CCD) [42, 45]. Previous studies showed that VesV2s and VesMa2 were isoallergens that significantly differed in the number of putative $N$-glycosylation sites (Table 3) [9, 22, 37, 40]. According to the sequencing analysis of VesT2s, it contains five $N$-glycosylation sites in VesT2a (Asn79, Asn99, Asn127, Asn187 and Asn325) and three $\mathrm{N}$-glycosylation sites in VesT2b (Asn1, Asn66 and Asn81). Based on this data, we speculated about a high degree of CCD. These data are potentially relevant, especially regarding to the cross-reaction $[40,46]$.

Via the turbidity method, $V$. tropica venom HAase was clearly active at a pH ranging from 2 to 5 (more than $80 \%$ of relative activity) with an optimal $\mathrm{pH}$ of approximately 3 to 4 . At pH 6 to 10, the activity reduced and no detectable activity was observed within the range of basic $\mathrm{pH}(8-10)$. Therefore, VesT2a was predicted as a strong acid HAase. However, the optimal pH (3 to 4) in this study was quite different from those of other wasp venoms, such as $V$. vulgaris ( $\mathrm{pH} 5-6), V$. germanica (pH 5-6) and D. maculata (pH 5-6) [47]. Generally, the

Table $3 \mathrm{~N}$-glycosylation in wasp venom HAase. Asn-Xaa-Ser/Thr residues represent the possible $\mathrm{N}$-glycosylation sites predicted by NetNGlyc 1.0 Server ( $\mathrm{N}$-glycosylation in $\mathrm{V}$. vulgaris and $\mathrm{V}$. magnifica HAase was obtained in the experiment in the native form)

\begin{tabular}{|c|c|c|c|c|}
\hline \multicolumn{2}{|c|}{ V. tropica (this study) } & \multicolumn{2}{|l|}{ V. vulgaris [22] } & \multirow{2}{*}{$\begin{array}{l}\text { V. magnifica } \\
\text { VesMa2 } \\
\text { (active HAase) }\end{array}$} \\
\hline $\begin{array}{l}\text { VesT2a (active } \\
\text { HAase) }\end{array}$ & $\begin{array}{l}\text { VesT2b (inactive } \\
\text { HAase) }\end{array}$ & $\begin{array}{l}\text { VesV2a (active } \\
\text { HAase) }\end{array}$ & $\begin{array}{l}\text { VesV2b } \\
\text { (inactive } \\
\text { HAase) }\end{array}$ & \\
\hline Asn79 & Asn1 & Asn79 & Asn66 & Asn105 \\
\hline Asn99 & Asn66 & Asn99 & Asn81 & Asn125 \\
\hline Asn127 & Asn81 & Asn127 & & Asn153 \\
\hline Asn187 & & & & Asn351 \\
\hline Asn325 & & & & \\
\hline
\end{tabular}


Table 4 The biochemical and physiological characterization of vespid venom

\begin{tabular}{lllll}
\hline Species & Molecular weight & $\mathrm{Pi}$ & Optimal pH & Reference \\
\hline D. maculate & 39 & $5-6$ & $5-6$ & {$[47]$} \\
V. germanica & 42 & $5-6$ & $5-6$ & {$[47]$} \\
V. vulgaris & 43 & $5-6$ & $5-6$ & {$[47]$} \\
V. tropica & $46-47$ & 8.91 & $3-4$ & Current study \\
P. paulista & 43.277 & 8.77 & $\mathrm{ND}$ & {$[9]$} \\
\hline
\end{tabular}

activity of HAases to degrade hyaluronic acid (HA) have an optimal $\mathrm{pH}$ ranging from 3 to 4 , which is in accordance with VesT2a in this study (Table 4) [48].

A previous study showed the high potency of $V$. tropica venom ( $\mathrm{PD}_{50} \sim 3 \mu \mathrm{g} / \mathrm{g}$ body weight of cricket) [26]. Venom HAase, a "spreading factor", is well-known for its toxin-enhancing activity. Therefore, the anti-HAase serum was produced. The anti-HAase serum shows neutralizing efficiency against crude venom by ratio the ratio of 1:12 (venom:antiserum). Inhibition of HAase activity not only prevents local tissue damage, but also retards the venom toxin diffusion into the tissues and blood circulation, resulting in the delay of fatal outcomes in several cases [13]. HAase activity may play a vital role in allergenicity and toxicity of venoms.

\section{Conclusions}

Hymenoptera venom showed cross-reactivity with bee and wasp venoms [2]. The allergic responses to wasp venom are known to cause life-threatening and fatal IgE-mediated anaphylactic reactions in sensitive individuals. The cross reactivity among the hyaluronidase from yellow jacket and bee venom are presumably induced by CCDs, but less often shared by peptide epitopes [19]. Knowledge on the structural determinants responsible for the allergic potency is expected to have important clinical implications.

\section{Funding}

This work was mainly supported by the Higher Education Research Promotion and National Research University (NRU) Project of Thailand, Office of the Higher Education Commission (CHE), through the Food and Functional Food Research Cluster of Khon Kaen University (KKU). It was also partially supported by the "The Thailand Research Fund - Master Research Granted (TRF-MAG)" year 2008 (MRG-WII515S069), "TRF-CHE jointly funded Research Grant for Mid-Career University Faculty", fiscal years 2007-2009; and KKU Research Fund, fiscal years 2007-2010.

\section{Authors' contributions}

PR conducted most of the experiments, coordinated the data analysis and drafted the manuscript. PI and SS contributed to bioinformatics analyses. NU conducted Western blotting experiments. SK contributed to the study design and writing of the manuscript. JD performed the molecular analyses and contributed to the writing of the manuscript. RP contributed to writing and editing of the manuscript. SR performed the proteomic study. SD designed the research and the experiments, coordinated the study, wrote and edited the manuscript. All authors read and approved the final manuscript.

\section{Competing interests}

The authors declare that there are no competing interests.

\section{Ethics approval and consent to participate}

The present study was approved by the Animal Ethics Committee of Khon Kaen University based on the Ethics for Animal Experimentation of the National Research Council of Thailand (reference. 0514.1.12.2/1).

\section{Author details}

'Protein and Proteomics Research Center for Commercial and Industrial Purposes (ProCCl), Department of Biochemistry, Faculty of Science, Khon Kaen University, Khon Kaen 40002, Thailand. 'Department of Chemistry, Faculty of Engineering, Rajamangala University of Technology Isan, Khon Kaen Campus, Khon Kaen, Thailand. ${ }^{3}$ Chulabhorn International College of Medicine, Thammasat University (Rangsit Campus), Pathumthani, Thailand. ${ }^{4}$ Division of Chemistry, Faculty of Science, Nakhon Phanom University, Nakhon Phanom, Thailand. ${ }^{5}$ Department of Clinical Chemistry, Faculty of Associated Medical Sciences, Khon Kaen University, Khon Kaen, Thailand. ${ }^{6}$ Genome Institute, National Center for Genetic Engineering and Biotechnology, National Science and Technology Development Agency (NSTDA), Pathumthani, Thailand. 'Division of Pharmacognosy and Toxicology, Faculty of Pharmaceutical Sciences, Khon Kaen University, Khon Kaen, Thailand.

Received: 20 April 2016 Accepted: 29 September 2016

Published online: 22 October 2016

\section{References}

1. King TP, Kochoumian $L$, Joslyn A. Wasp venom proteins: phospholipase A1 and B. Arch Biochem Biophys. 1984;230(1):1-12.

2. King TP, Lu G, Gonzalez M, Qian N, Soldatova L. Yellow jacket venom allergens, hyaluronidase and phospholipase: sequence similarity and antigenic crossreactivity with their hornet and wasp homologs and possible implications for clinical allergy. J Allergy Clin Immunol. 1996;98(3):588-600.

3. Jalaei J, Fazeli M, Rajaian H, Shekarforoush SS. In vitro antibacterial effect of wasp (Vespa orientalis) venom. J Venom Anim Toxins Incl Trop Dis. 2014; 20(22):1-6.

4. Santos LD, Pieroni M, Menegasso ARS, Pinto JRAS, Palma MS. A new scenario of bioprospecting of Hymenoptera venoms through proteomic approach. J Venom Anim Toxins Incl Trop Dis. 2011;17:364-77.

5. King TP, Alagon AC, Kuan J, Sobotka AK, Lichtenstein LM. Immunochemical studies of yellowjacket venom proteins. Mol Immunol. 1983;20(3):297-308.

6. Abe T, Sugita M, Fujikura T, Hiyoshi J, Akasu M. Giant hornet (Vespa mandarinia) venomous phospholipases. The purification, characterization and inhibitory properties by biscoclaurine alkaloids. Toxicon. 2000;38(12):1803-16.

7. Kreil G. Hyaluronidases - a group of neglected enzymes. Protein Sci. 1995; 4(9):1666-9.

8. Sukprasert S, Rungsa P, Uawonggul N, Incamnoi P, Thammasirirak S, Daduang J, et al. Purification and structural characterisation of phospholipase A1 (Vespapase, Ves a 1) from Thai banded tiger wasp (Vespa affinis) venom. Toxicon. 2013;61:151-64.

9. Justo Jacomini DL, Campos Pereira FD, Santos Pinto JR A d, dos Santos LD, da Silva Neto AJ, Giratto DT, et al. Hyaluronidase from the venom of the social wasp Polybia paulista (Hymenoptera, Vespidae): cloning, structural modeling, purification, and immunological analysis. Toxicon. 2013;64:70-80.

10. Bordon KCF, Perino MG, Giglio JR, Arantes EC. Isolation, enzymatic characterization and antiedematogenic activity of the first reported rattlesnake hyaluronidase from Crotalus durissus terrificus venom. Biochimie. 2012;94(12):2740-8.

11. Feng L, Gao R, Gopalakrishnakone P. Isolation and characterization of a hyaluronidase from the venom of Chinese red scorpion Buthus martensi. Comp Biochem Physiol C Toxicol Pharmacol. 2008;148(3):250-7.

12. Girish KS, Shashidharamurthy R, Nagaraju S, Gowda TV, Kemparaju K. Isolation and characterization of hyaluronidase a "spreading factor" from Indian cobra (Naja naja) venom. Biochimie. 2004;86(3):193-202.

13. Wahby AF, Mahdy e-SM, El-Mezayen HA, Salama WH, Abdel-Aty AM, Fahmy AS. Egyptian horned viper Cerastes cerastes venom hyaluronidase: Purification, partial characterization and evidence for its action as a spreading factor. Toxicon. 2012;60(8):1380-9. 
14. Magalhães MR, da Silva Jr NJ, Ulhoa CJ. A hyaluronidase from Potamotrygon motoro (freshwater stingrays) venom: isolation and characterization. Toxicon. 2008;51(6):1060-7.

15. Nagaraju S, Devaraja S, Kemparaju K. Purification and properties of hyaluronidase from Hippasa partita (funnel web spider) venom gland extract. Toxicon. 2007:50(3):383-93.

16. Hoffman DR, Dove DE, Jacobson RS. Allergens in Hymenoptera venom: XX. Isolation of four allergens from imported fire ant (Solenopsis invicta) venom. J Allergy Clin Immunol. 1988;82(5 Pt 1):818-27.

17. Xia X, Liu R, Li Y, Xue S, Liu Q, Jiang X, et al. Cloning and molecular characterization of scorpion Buthus martensi venom hyaluronidases: a novel full-length and diversiform noncoding isoforms. Gene. 2014;547(2):338-45.

18. Kaneiwa T, Mizumoto S, Sugahara K, Yamada S. Identification of human hyaluronidase-4 as a novel chondroitin sulfate hydrolase that preferentially cleaves the galactosaminidic linkage in the trisulfated tetrasaccharide sequence. Glycobiology. 2010;20(3):300-9.

19. Jin C, Focke M, Léonard R, Jarisch R, Altmann F, Hemmer W. Reassessing the role of hyaluronidase in yellow jacket venom allergy. J Allergy Clin Immunol. 2010;125(1):184-90. e1.

20. Kolarich D, Leonard R, Hemmer W, Altmann F. The N-glycans of yellow jacket venom hyaluronidases and the protein sequence of its major isoform in Vespula vulgaris. FEBS J. 2005;272(20):5182-90.

21. Justo Jacomini DL, Gomes Moreira SM, Campos Pereira FD, de Zollner RL, Brochetto Braga MR. Reactivity of IgE to the allergen hyaluronidase from Polybia paulista (Hymenoptera, Vespidae) venom. Toxicon. 2014;82:104-11.

22. Seppälä U, Selby D, Monsalve R, King TP, Ebner C, Roepstorff P, et al. Structural and immunological characterization of the $\mathrm{N}$-glycans from the major yellow jacket allergen Ves $\vee 2$ : The $\mathrm{N}$-glycan structures are needed for the human antibody recognition. Mol Immunol. 2009:46(10):2014-21.

23. Arming $\mathrm{S}$, Strobl B, Wechselberger $\mathrm{C}$, Kreil G. In vitro mutagenesis of $\mathrm{PH}-20$ hyaluronidase from human sperm. Eur J Biochem. 1997;247(3):810-4.

24. Jin C, Hantusch B, Hemmer W, Stadlmann J, Altmann F. Affinity of IgE and IgG against cross-reactive carbohydrate determinants on plant and insect glycoproteins. J Allergy Clin Immunol. 2008;121(1):185-90. e2.

25. King TP, Joslyn A, Kochoumian L. Antigenic cross-reactivity of venom proteins from hornets, wasps, and yellow jackets. J Allergy Clin Immunol. 1985;75(5):621-8.

26. Rungsa P, Incamnoi P, Sukprasert P, Uawonggul N, Klaynongsruang S, Daduang J, et al. Comparative proteomic analysis of two wasps venom, Vespa tropica and Vespa affinis. Toxicon. 2016;119:159-67.

27. Schmidt JO, Yamane S, Matsuura M, Starr CK. Hornet venoms: lethalities and lethal capacities. Toxicon. 1986;24(9):950-4.

28. Gawade SP. The effect of venom from the Indian tropical wasp Vespa tropica on nerve - muscle preparations from Drosophila larvae. Toxicon. 1983; 21(6):882-6

29. Incamnoi P, Patramanon R, Thammasirirak S, Chaveerach A, Uawonggul N, Sukprasert S, et al. Heteromtoxin (HmTx), a novel heterodimeric phospholipase A2 from Heterometrus laoticus scorpion venom. Toxicon. 2013;61:62-71.

30. Saitou N, Nei M. The neighbor-joining method: a new method for reconstructing phylogenetic trees. Mol Biol Evol. 1987;4(4):406-25.

31. Bordoli L, Kiefer F, Arnold K, Benkert P, Battey J, Schwede T. Protein structure homology modeling using SWISS-MODEL workspace. Nat Protoc. 2009;4:1-13.

32. Arnold K, Bordoli L, Kopp J, Schwede T. The SWISS-MODEL workspace: a web-based environment for protein structure homology modelling. Bioinformatics. 2006;22(2):195-201.

33. Mio K, Stern R. Reverse hyaluronan substrate gel zymography procedure for the detection of hyaluronidase inhibitors. Glycoconj J. 2000;17(11):761-6.

34. Pukrittayakamee S, Warrell DA, Desakorn V, McMichael AJ, White NJ, Bunnag D. The hyaluronidase activities of some Southeast Asian snake venoms. Toxicon. 1988;26(7):629-37.

35. Kolarich D, Loos A, Leonard R, Mach L, Marzban G, Hemmer W, et al. A proteomic study of the major allergens from yellow jacket venoms. Proteomics. 2007;7(10):1615-23.

36. Marković-Housley Z, Miglierini G, Soldatova L, Rizkallah PJ, Müller U, Schirmer T. Crystal Structure of hyaluronidase, a major allergen of bee venom. Structure. 2000;8(10):1025-35

37. Skov LK, Seppala U, Coen JJ, Crickmore N, King TP, Monsalve R, et al. Structure of recombinant Ves $\vee 2$ at 2.0 Angstrom resolution: structural analysis of an allergenic hyaluronidase from wasp venom. Acta Crystallogr D Biol Crystallogr. 2006;62(Pt 6):595-604.

38. Lu G, Kochoumian L, King TP. Sequence identity and antigenic cross-reactivity of white face hornet venom allergen, also a hyaluronidase, with other proteins. J Biol Chem. 1995;270(9):4457-65.

39. Pinto JR, Santos LD, Arcuri HA, Dias NB, Palma MS. Proteomic characterization of the hyaluronidase (E.C. 3.2.1.35) from the venom of the social wasp Polybia paulista. Protein Pept Lett. 2012;19(6):625-35.

40. An S, Chen L, Wei JF, Yang X, Ma D, Xu X, et al. Purification and characterization of two new allergens from the venom of Vespa magnifica. PLoS One. 2012:7(2):e31920.

41. El-Safory NS, Fazary AE, Lee C-K. Hyaluronidases, a group of glycosidases: current and future perspectives. Carbohydr Polym. 2010;81(2):165-81.

42. King TP, Spangfort MD. Structure and biology of stinging insect venom allergens. Int Arch Allergy Immunol. 2000;123(2):99-106.

43. King TP, Wittkowski KM. Hyaluronidase and hyaluronan in insect venom allergy. Int Arch Allergy Immunol. 2011;156(2):205-11.

44. Seismann H, Blank S, Braren I, Greunke K, Cifuentes L, Grunwald T, et al. Dissecting cross-reactivity in hymenoptera venom allergy by circumvention of a-1,3-core fucosylation. Mol Immunol. 2010;47(4):799-808.

45. Hemmer W, Focke M, Kolarich D, Dalik I, Gotz M, Jarisch R. Identification by immunoblot of venom glycoproteins displaying immunoglobulin E-binding $\mathrm{N}$-glycans as cross-reactive allergens in honeybee and yellow jacket venom. Clin Exp Allergy. 2004;34(3):460-9.

46. Hemmer W, Focke M, Kolarich D, Wilson IBH, Altmann F, Wöhrl S, et al. Antibody binding to venom carbohydrates is a frequent cause for double positivity to honeybee and yellow jacket venom in patients with stinginginsect allergy. J Allergy Clin Immunol. 2001;108(6):1045-52.

47. Girish KS, Kemparaju K. The magic glue hyaluronan and its eraser hyaluronidase: a biological overview. Life Sci. 2007;80(21):1921-43.

48. Hokputsa S, Jumel K, Alexander C, Harding SE. Hydrodynamic characterisation of chemically degraded hyaluronic acid. Carbohydr Polym. 2003;52(2):111-7.

\section{Submit your next manuscript to BioMed Central and we will help you at every step:}

- We accept pre-submission inquiries

- Our selector tool helps you to find the most relevant journal

- We provide round the clock customer support

- Convenient online submission

- Thorough peer review

- Inclusion in PubMed and all major indexing services

- Maximum visibility for your research

Submit your manuscript at www.biomedcentral.com/submit 